\title{
A comparative study of the elastic properties of continuous tear curvilinear capsulorhexis versus capsulorhexis produced by radiofrequency endodiathermy
}

\author{
J Luck, A K Brahma, B A Noble
}

\begin{abstract}
Capsulorhexis using radio-frequency endodiathermy may confer some advantages over continuous tear curvilinear capsulorhexis (CTCC) in certain clinical situations. It is unclear whether a capsulorhexis produced in this fashion has the clinically advantageous elasticity and resistance to tearing that a CTCC has been demonstrated to have. To investigate this, a test of capsular elasticity was carried out on pairs of eyes obtained from an eye bank, 42 eyes of 21 patients in total, using modified digital vernier calipers. One eye of each pair had a CTCC, the other a diathermy capsulorhexis (DC). The elasticity of the capsule in both groups was expressed by comparing the circumference of the capsulotomy at rest with its circumference at rupture. The mean capsular elasticity of the CTCC group was significantly greater than that of the DC group $(p<<0.001)$. The capsular edge in both groups was examined using scanning electron microscopy, and the difference in morphology appears to be the source of the difference in elasticity.

(Br F Ophthalmol 1994; 78: 392-396)
\end{abstract}

Continuous tear curvilinear capsulorhexis (CTCC), as independently described and developed by Gimbel and Neuhann, ${ }^{1}$ is considered by some authorities to be a prerequisite to safe endocapsular phacoemulsification. The method is also applicable to extracapsular extraction, using modified techniques such as hydro- or viscoexpression for nuclear extraction. ${ }^{234}$

Capsular surgery using radiofrequency endodiathermy (RFE) may confer some advantages over CTCC in particular clinical situations. The method was originally described by Klöti in $1984,^{5}$ and further by Gassmann and colleagues in $1988 .{ }^{6}$ The capsule is coagulated and cut by the platinum alloy tip of a probe, heated to approximately $160^{\circ} \mathrm{C}$ by a modulated high frequency current of $500 \mathrm{kHz}$. The technique does not rely on the presence of a red reflex, and is easy to perform. It enables a capsulorhexis to be performed on the infantile capsule, which is highly elastic and difficult to tear, and may be used in small pupil cases where CTCC is hazardous. Although capsular diathermy is commercially available and in current clinical use, we are unaware of any studies that have addressed the question of whether capsulorhexis produced using the two different methods differ in elastic properties, and subsequently in clinical performance.
In order to investigate this question, we carried out a study to compare the deformation properties and resistance to radial tear formation of capsulorhexis produced by a tearing method and that produced using diathermy. The method was very similar, with minor modifications, to that used in previous studies of the elastic properties of CTCC. ${ }^{27}$

\section{Materials and methods}

We obtained eyes in pairs from an eye bank, with corneoscleral discs removed. Any pair with either or both eyes having unacceptable damage resulting from corneal retrieval or other obvious morphological changes were discarded. A total of 42 eyes from 21 patients (14 males and eight females) were studied. The mean age of the patients was 69 years (range 18 to 85 years). The average time post mortem to the eyes being received was 2 days, with a maximum of 3 days. In order to match as far as possible for age, sex, time interval following death before retrieval, and the time interval between retrieval and examination, one eye of each pair was assigned to each of two groups, group 1 and group 2.

In both groups, the iris was removed, permitting 'open-sky' access to the anterior capsule. The eyes from group 1 had a CTCC performed, and in those from group 2 a diathermy capsulorhexis (DC) was performed. The capsular surgery in both groups took place under a viscoelastic obtained as waste from routine cataract surgery. The CTCC was performed using a cystotome to initiate the tear, and subsequently completed using Uttrata's forceps. In both groups an attempt was made to keep the capsulorhexis circular and of approximately 4 to $5 \mathrm{~mm}$ in diameter. The nucleus in both groups was delivered by hydrodissection followed by hydroexpression. Any eyes in which radial tear formation occurred during nucleus delivery were noted. This was done in order to study any difference in the rate of radial tear formation resulting from nuclear expression between the two groups, and was necessary because the stretch test used (see below) required an intact capsulotomy. All eyes had nuclear removal carried out; capststes with radial tears were excluded along with their fellow eye, in order to keep the data as paired data and to minimise possible bias. In total, 34 eyes from 17 patients were entered into the stretch test phase of the study.

The stretch test was performed using digital vernier calipers (Mitutoyo; accuracy $0.01 \mathrm{~mm}$ ), modified by attaching pins to the measuring arms, allowing introduction into the capsular 


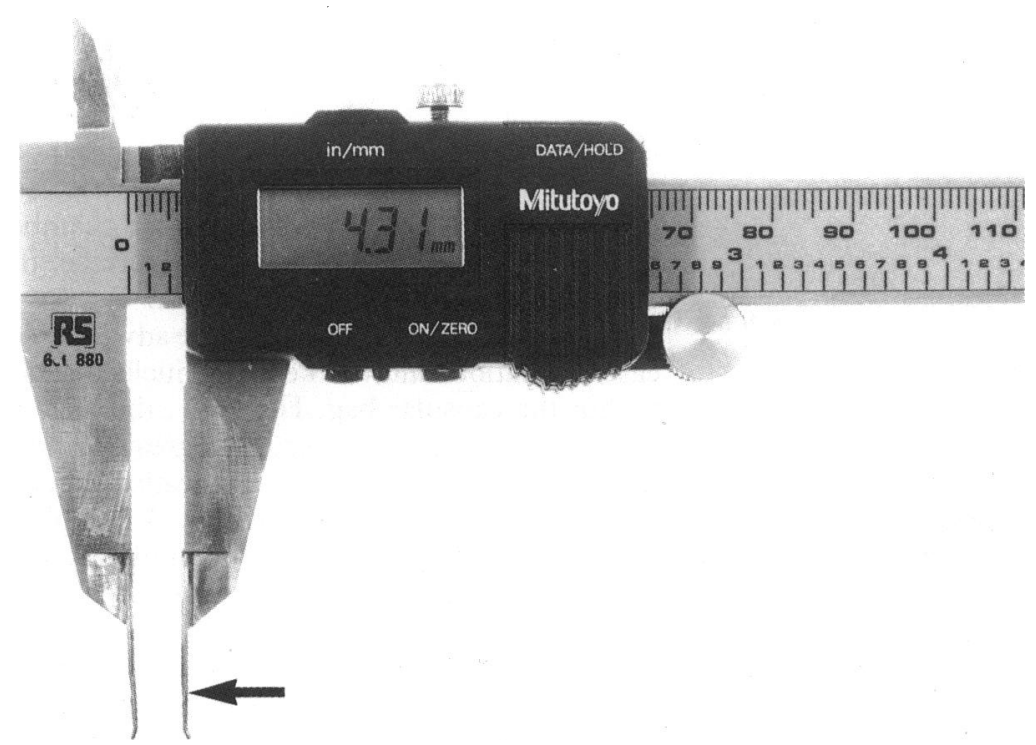

Figure 1 Modified digital vernier calipers. Pins have been attached to the measuring arms (arrow) to allow introduction into the capsular opening.
Figure 2 The measuring arms have been introduced into the capsulorhexis, and opened, stretching the capsule. The capsule capsule. The capsule, configuration before rupture, allowing simple calculation of its circumference. The capsular edge is clearly seen (arrows). opening (Fig 1). The calipers were then used to measure the diameter of the capsulorhexis in both the vertical and horizontal planes.

The calipers were then gently opened until the capsule was deformed into a 'racetrack' configuration, with straight edges (Fig 2). The capsule then stretched, and ultimately ruptured. At the point of rupture, the readout on the digital scale, in millimetres, was noted.

The circumference of the capsulorhexis at rest $\left(C_{\text {rest }}\right)$ in all eyes was calculated as follows:

$$
\begin{aligned}
& \mathrm{C}_{\text {rest }}=\pi \mathrm{D} \\
& (\mathrm{D}=\text { diameter of capsulorhexis })
\end{aligned}
$$

The circumference of the capsulorhexis at rupture $\left(\mathrm{C}_{\text {rupt }}\right)$ was calculated as follows:

$$
\begin{aligned}
& \mathrm{C}_{\text {rupt }}=2 \mathrm{R}_{\text {cal }}+\pi \mathrm{d} \\
& \left(\mathrm{R}_{\mathrm{cal}}=\text { caliper reading at rupture, } \mathrm{d}=\right.\text { diameter }
\end{aligned}
$$$$
\text { of the caliper pins) }
$$

$\pi \mathrm{d}$ is an expression of the total arc of contact between the pins and the capsule. As the pins were $1 \mathrm{~mm}$ in diameter, this value was equal to $\pi$.

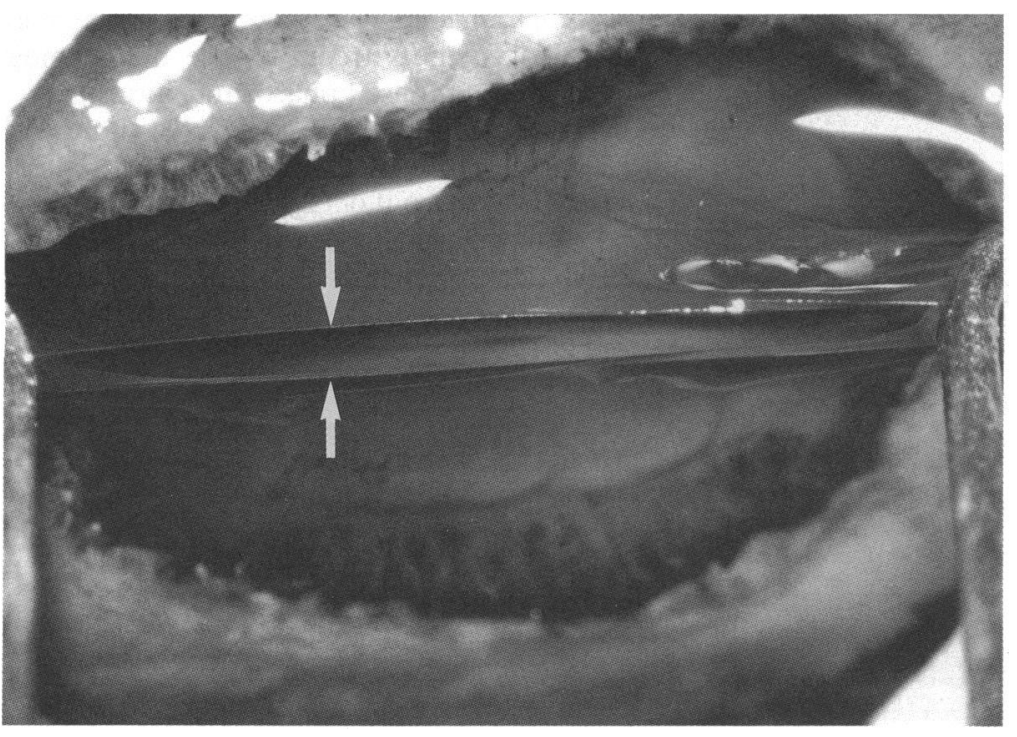

An expression for the stretching capacity of the capsule in both groups was derived by comparing the circumference of the capsulotomy at rest to its circumference at rupture. This was expressed as a percentage - for example, if a capsule measured $15 \mathrm{~mm}$ in circumference at rest and $22.5 \mathrm{~mm}$ at rupture, then the capsule would have stretched by $50 \%$ from its original dimensions.

Specimens of anterior lens capsule from both groups were obtained for examination using scanning electron microscopy (SEM). Owing to the difficulties in preparation of loose capsule, the whole lens with the anterior capsulectomy performed was prepared. The tissue was prepared for SEM by fixing for 1 hour at $4^{\circ} \mathrm{C}$ in an isotonic $2 \cdot 5 \%$ glutaraldehye, $2 \%$ formaldehyde fixative in a sodium phosphate buffer, and then post-fixed with $1.0 \%$ osmium tetroxide for structural stability. The specimens were then dehydrated in acetone, critical point dried, and sputter coated with gold.

\section{Results}

The average dimensions of the capsular openings in group 1 was $5.4 \mathrm{~mm}$ (range $4.4 \mathrm{~mm}$ to $6.6 \mathrm{~mm}$ ), that in group 2 being $5.5 \mathrm{~mm}$ (range $4.5 \mathrm{~mm}$ to $6.1 \mathrm{~mm}$ ). In neither group did the diameter in the horizontal direction differ from that in the vertical direction by more than $1.7 \mathrm{~mm}$, the average discrepancy in the CTCC group being $0.5 \mathrm{~mm}$, that in the DC group being $0.4 \mathrm{~mm}$. There was a significant difference in the incidence of radial tear formation on nuclear expression between the two groups. Four eyes in group 2, the DC group, had radial tear formation, whereas no radial tears occurred in the CTCC group, group 1 (Fisher's exact test; $\mathrm{p}<0.05)$. Therefore eight eyes in total, four eyes from the DC group and their fellow four eyes from the CTCC group, were excluded from the stretch test for the reasons stated above.

The mean elasticity of the capsulorhexis in the CTCC group was $61 \%$ (range $41 \%$ to $82 \%$ ), compared with the mean elasticity in the DC group of $36 \%$ (range $11 \%$ to $50 \%$ ). There was a highly significant difference between the elasticity of the capsulorhexis in the two groups (Fig 3 ); (paired $t$ test; $t=6 \cdot 10 ; \mathrm{p} \ll 0 \cdot 001$ ).

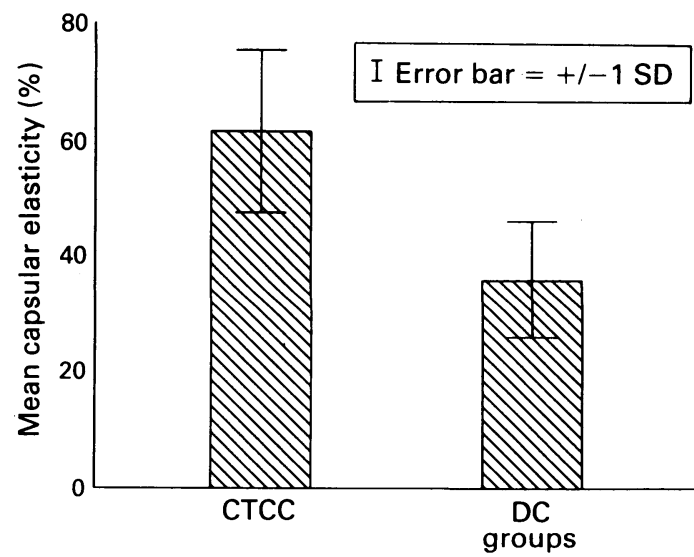

Figure 3 Capsular elasticity: mean elasticity of the continuous tear curvilinear capsulorhexis (CTCC) group versus mean elasticity of the diathermy capsulorhexis $(D C)$ group. 
Figure 4 Relation between the diameter of the capsulorhexis at rest and the circumference at rupture for both groups. The correlation in the diathermy capsulorhexis (DC) group does not reach significance. For the continuous curvilinear capsulorhexis (CTCC) group, $\mathrm{r}=0.59$ $(p<0.01)$; for the DC group, $\mathrm{r}=0.45(p>0.05)$.

Figure 5 Scanning electron micrograph of the capsular edge following CTCC. The smooth capsular edge is demonstrated (arrows). The capsule $(C)$ has artefactually separated from the cuboidal lens epithelium $(E)$. Lens fibres $(F)$ are seen in the foreground. (Bar=200 $\mu \mathrm{m}$.)

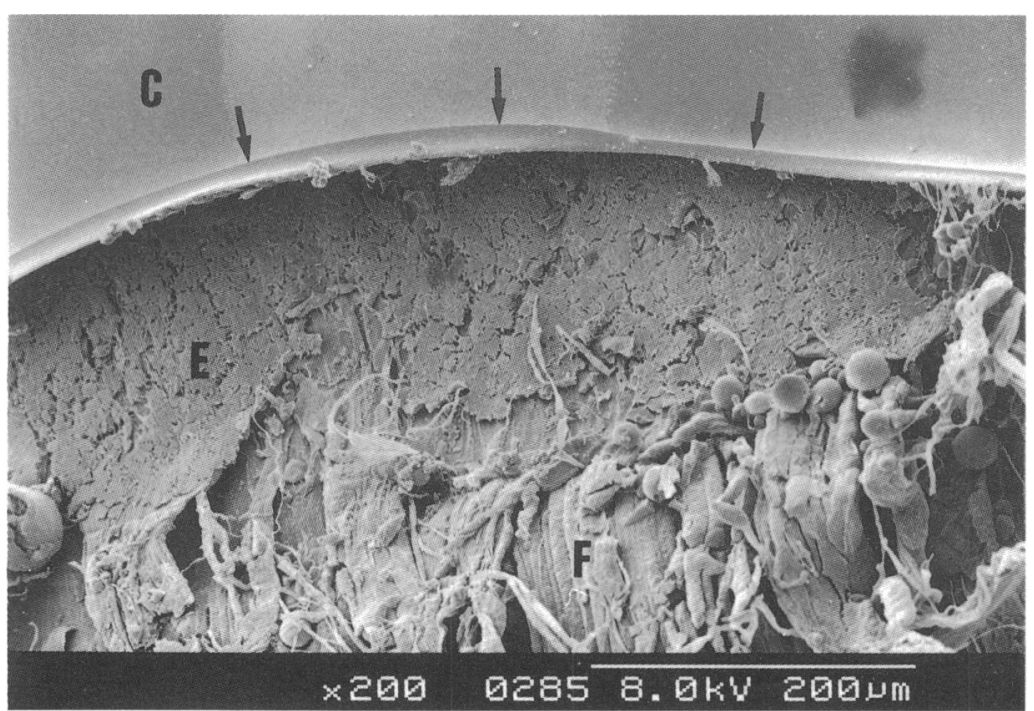

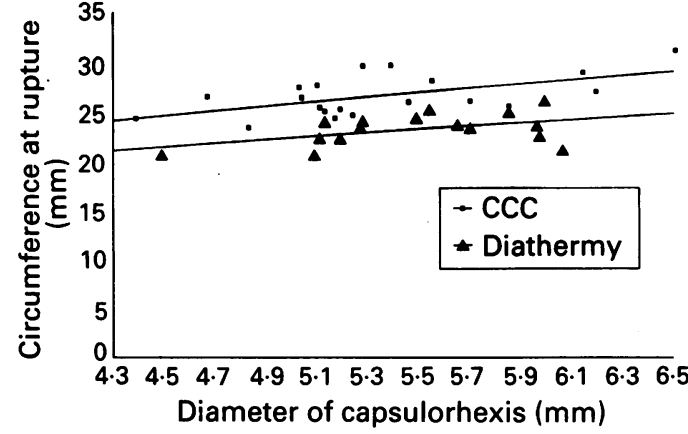

There was a significant correlation between the circumference of the capsulorhexis at rest and the circumference at rupture for the CTCC group $(r=0.59 ; \mathrm{p}<0.01)$. There was no significant correlation between these measurements in the diathermy group (Fig 4). There was no significant correlation between the age or sex of the patient and the capsular elasticity in either group.

SEM examination revealed a marked difference between the two specimens. The CTCC specimen showed a very fine edge, with no evidence at fairly low power of any defects in it (Fig 5). Under higher magnification, the CTCC edge is seen to be completely smooth and free from irregularity apart from some cellular debris. The lamellar organisation of the collagen in the capsule was demonstrated in this view (Fig 6). The DC specimen showed an extremely abnormal and disrupted capsular edge. At low power, the edge was seen to be highly irregular, with some of the anterior lens fibres becoming adherent to the capsular edge (Fig 7). From a different part of the specimen, the disorganised nature of the DC capsular edge was again seen ( Fig 8), and showed loss of the normal lamellar architecture of the capsule, with distorted and unrecognisable collagen fibrils and adherent lens fibres.

\section{Discussion}

CTCC is a surgical skill which can be demanding. An opening with an intact edge and near circular dimensions, to eliminate weak spots, is desirable.
The ideal size of a capsulorhexis is a debatable issue. Tearing a large capsulorhexis runs the risk of zonular engagement and peripheral tear extension, and if peroperative miosis were to occur, the view of the capsular edge may be lost, and accurate capsular implantation jeopardised. In the presence of a large capsulorhexis, the nucleus will tend to dislocate into the anterior chamber during phacoemulsification, and increase the chance of iris and endothelial trauma.

Small capsular openings have the advantage of ease of creation, and will keep the nucleus firmly within the capsular bag. However, the edge of the capsulorhexis is exposed to a greater risk of damage with the phacoemulsification probe, leading to possible tear formation. A complication of capsulorhexis that is becoming increasingly recognised is progressive capsular fibrosis, where the anterior capsule contracts postoperatively leading to a small round opening. This may occur in a capsulorhexis of any size, but is more problematical if the original opening is small. The capsule rarely encroaches on the visual axis, but the reduction in the view of the peripheral retina can be a major problem in patients requiring laser treatment or with peripheral retinal pathology. Some authors have described techniques whereby an initial small capsulorhexis, with the advantages listed above, is converted to a larger opening once lens implantation has been carried out. ${ }^{8}$

Radial tears of the edge of a capsulorhexis may occur during its creation, during emulsification of the nucleus, or during aspiration of cortical lens material. The stages where this is particularly likely to occur are during sculpting of a hard nucleus, causing excessive nuclear movement, during nucleofractis, or when reaching for lens matter in the capsular fornix with the irrigation aspiration probe. Tears are more likely to occur in situations where there is relative anterior encroachment of the zonules' or where there is positive vitreous pressure. Although radial tears tend to be prevented from propagation by the zonules in ordinary 'envelope' type capsulotomy, ${ }^{10} \mathrm{a}$ radial tear before or during a phacoemulsification procedure increases the chances of zonular and posterior capsular rupture, owing to the relatively greater distorting forces involved. A posterior capsular rupture with remaining nuclear fragments which may dislocate into the vitreous is one of the most feared complications of phacoemulsification.

Small incision surgery can involve the use of small optic intraocular lenses, which require accurate centration if debilitating optical aberrations are to be avoided. In the presence of a radial tear, lens centration may be compromised, either because of unequal capsular fibrosis and shrinkage postoperatively, or by escape of one haptic from the capsular bag ('pea pod' effect)." Because of these factors, some authorities recommend that if a capsulorhexis rim is not intact for any reason, then phacoemulsification should be abandoned, and conversion to extracapsular extraction with larger optic intraocular lens should take place.

Radiofrequency endodiathermy was developed in order to try and overcome some of the difficulties inherent in creating an intact circular 


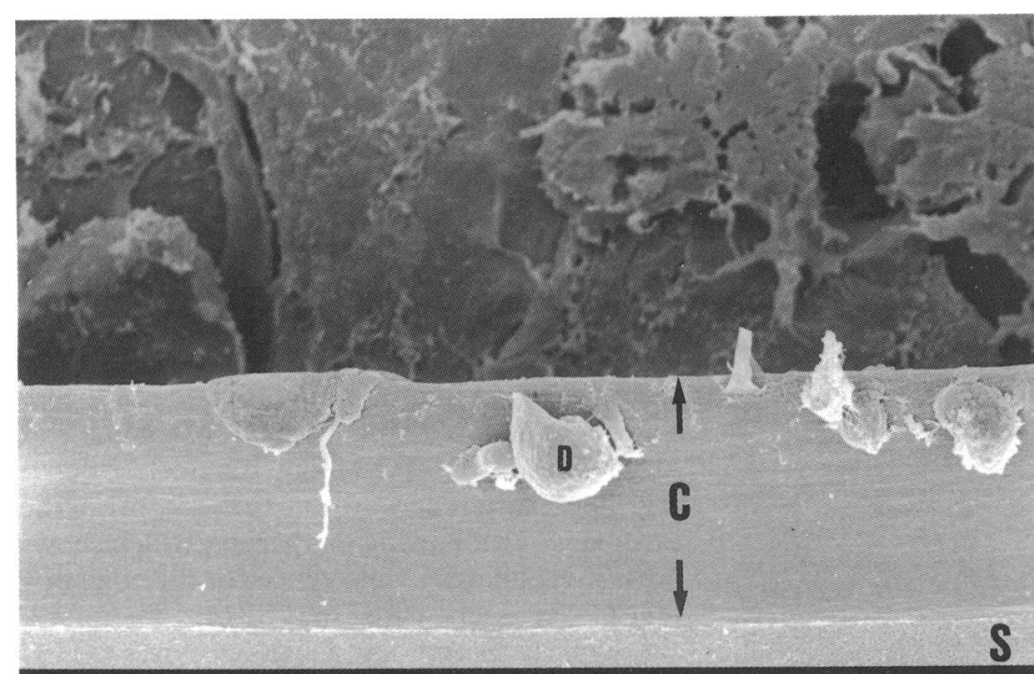

\section{$\times 1.5 \mathrm{k} 02828 . \overline{\mathrm{kV}} 20 \mathrm{~mm}$}

Figure 6 Scanning electron micrograph of the capsular edge following CTCC. At this higher magnification, the very smooth edge of the capsulorhexis $(C)$ is seen (between arrows) just above the capsular surface $(S)$. There is a small amount of cellular debris on the capsulorhexis edge (D). (Bar=20 um.)

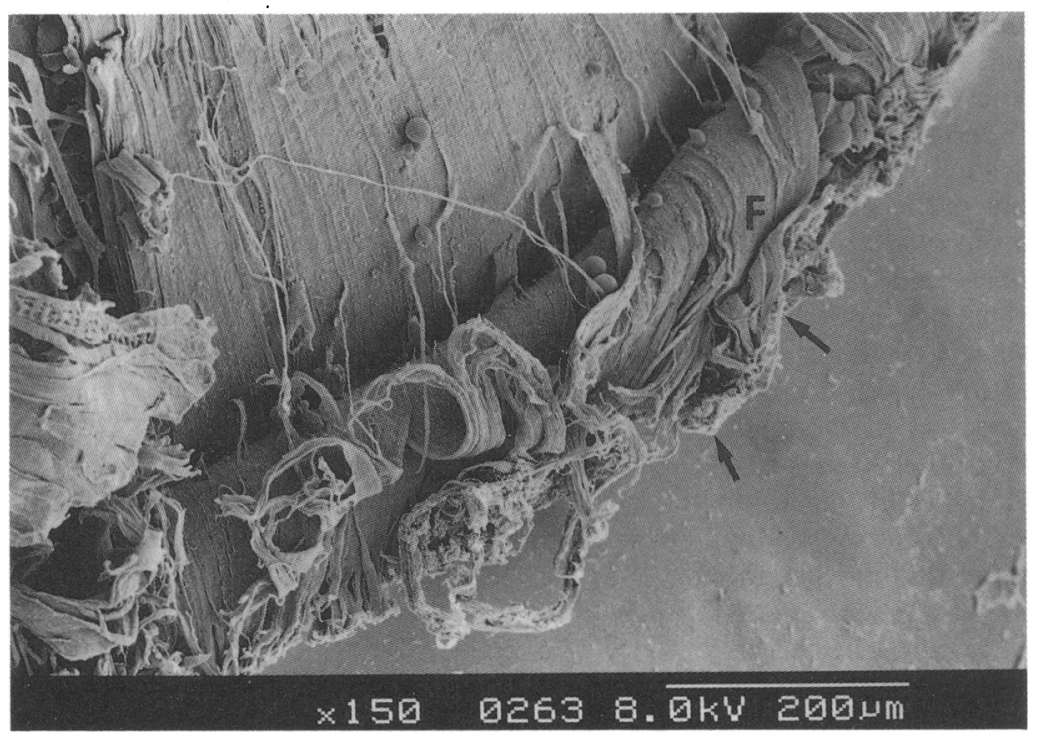

Figure 7 Scanning electron micrograph of the capsular edge following diathermy. The capsular edge (arrows) is distorted and abnormal, and there has been coagulation and bonding of the edge to the anterior lens fibres $(F)$. (Bar=200 $\mu \mathrm{m}$.

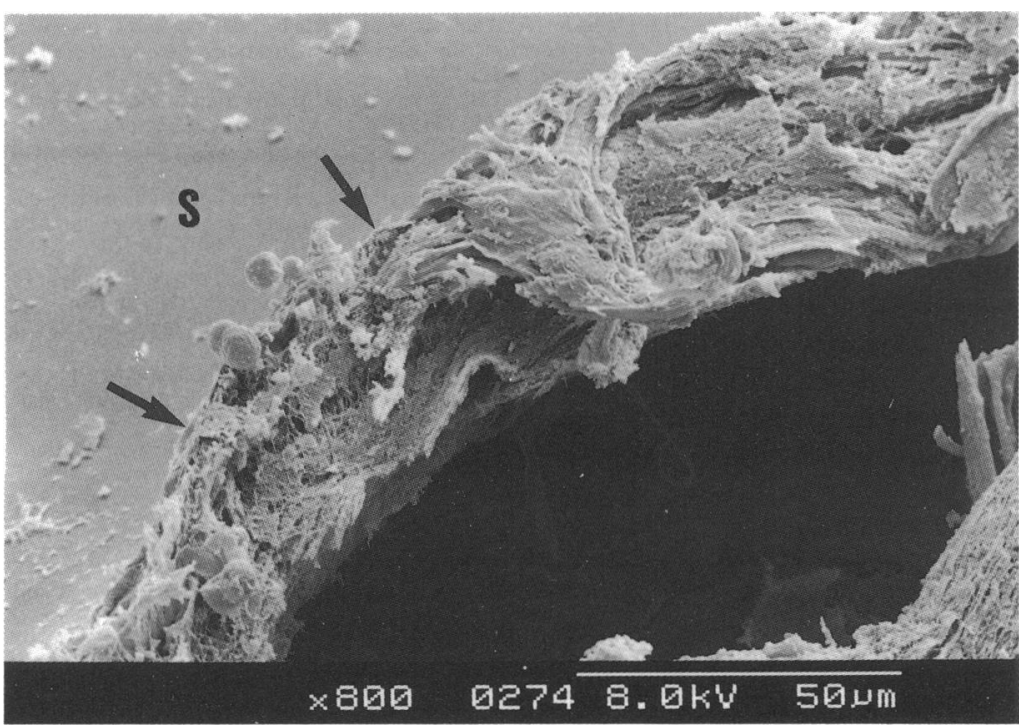

Figure 8 Scanning electron micrograph of the capsular edge following a diathermy capsulorhexis. This view from a different part of the same specimen as Figure 7 shows the distortion and coagulation of the capsular edge (arrows), adjacent to the capsular surface (S). (Bar=50 $\mu \mathrm{m}$. capsular opening of a planned size. It appears to have advantages over CTCC in certain situations. For example, a DC is easy to perform in any situation, regardless of the quality of the red reflex. It is possible to create a highly predictable capsulorhexis even in cases with mature lens opacities. In contrast, a poor red reflex increases considerably the difficulty of creating an optimal CTCC. The juvenile capsule is highly elastic and difficult to tear, and diathermy enables a controlled opening to be created in these situations. Diathermy may be used unseen behind the iris in small pupil cases, and although CTCC may be performed under these circumstances, it is an advanced surgical skill with some danger. Despite these advantages, the major consideration must be the elasticity and strength of the capsular opening.

The elastic properties of the lens capsule and in particular circular capsular openings, are well known and have been the subject of several studies. $^{271213}$ Assia and colleagues showed that a CTCC can be enlarged by approximately $0.6 \mathrm{~mm}$ for every $1.0 \mathrm{~mm}$ of capsulorhexis circumference, representing a $60 \%$ 'elasticity' of the capsular opening. ${ }^{7}$ Our findings are in accordance with this. However, the DC appears to be significantly less elastic than a CTCC, with an average $36 \%$ elasticity. This would suggest that capsular surgery using diathermy should be restricted to cases where this disadvantage is outweighed by the advantages cited above.

There was a significant difference in the rate of capsular tearing following nuclear expression between the two groups. No eyes from the CTCC group had radial tear formation on nuclear removal. This finding supports the accumulating evidence that a CTCC is fully compatible with nuclear expression, providing techniques using hydraulic pressure (either with balanced salt or a viscoelastic ${ }^{314}$ ) are used. Therefore, there appears to be an increased risk of tearing of a diathermy capsulorhexis following nuclear removal.

The correlation between the circumference of the CTCC at rest and when stretched to its rupture point has been previously reported in a study of the elastic properties of CTCC. ${ }^{7}$ Our method differed slightly in that we deliberately clustered our capsulorhexis sizes around those desired clinically, but despite this we have still demonstrated a significant linear correlation. A CTCC therefore has elastic properties which do not depend on its original size; in other words a small capsulorhexis has similar elastic behaviour to a larger one.

We postulate that the difference in elasticity between the groups, and possibly also the lack of correlation between the 'at rest' and 'rupture' circumference in the diathermy group, is due to the microscopic anatomy of the capsular edge. The capsule is made up of a series of collagen lamellae that run parallel to the surface and at right angles to the zonular insertions. ${ }^{15}$ Recent evidence suggests a more complex arrangement of a densely packed, three dimensional branching network of fine microfibrils, when examined at very high magnification using specialised preparation techniques. ${ }^{16}$ This lamellar arrangement can be seen in the edge of the capsule in our 


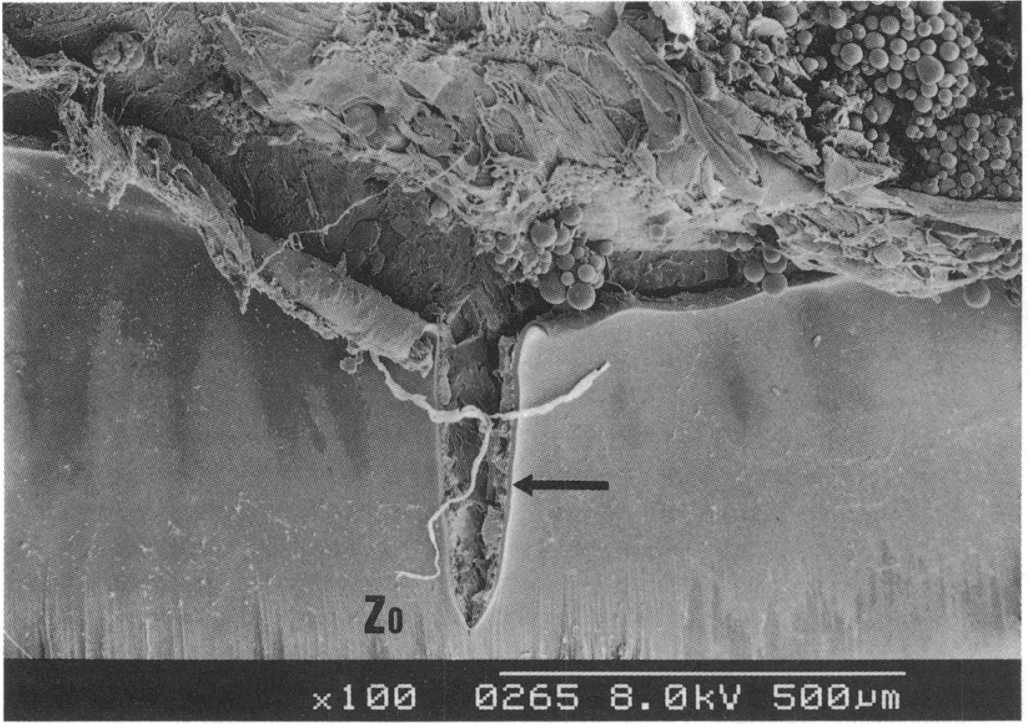

Figure 9 Scanning electron micrograph of the capsular edge following a diathermy capsulorhexis. A radial tear, approximately $350 \mathrm{\mu m}$ in length has formed (arrow) and extends peripherally to the zonular insertions (Zo). $($ Bar $=500$ um.)

CTCC specimen (Fig 6), a finding previously illustrated in a capsulorhexis specimen by Straatsma and coworkers. ${ }^{17} \mathrm{~A}$ tear in the capsule therefore presumably runs naturally in a curvilinear fashion along these natural fibrillar 'fault lines', and will obey vector forces induced by the surgeon when doing so. The elasticity of the lens capsule is probably partly due to this ultrastructure, and also to the type of collagen found in the capsule. The basement membrane of the capsule is primarily composed of type IV collagen, although recent work has demonstrated the presence of types I and III collagen, ${ }^{18}$ making it unique among ocular basement membranes. Type III collagen is found in other tissues that exhibit elastic properties. Further evidence for the capsular ultrastructure contributing to the elasticity of the capsule is the finding that the fibrillar ultrastructure is less marked with age, as is the elastic quality of the capsule, the former perhaps being a morphological manifestation of the latter. ${ }^{19}$

Diathermy capsulorhexis, where the cutting process relies on generated heat, will not respect the natural arrangement of the collagen fibrils in the capsule. This is evident on examination of the SEM specimens. Most conspicuous is the general irregularity compared with the CTCC specimen (Figs 7 and 8). This leads to an edge with areas of differing resistance to breakage, and force applied to this causes rupture at the weakest point, and a radial tear will form. This is illustrated in one section of the DC specimen examined by SEM where a small radial tear, some $350 \mu \mathrm{m}$ in length, has formed and is extending peripherally towards the zonular insertions (Fig 9). The irregularity of the edge may be exacerbated by fluctuations in diathermy current that occur if a build up of debris occurs on the probe tip, partially insulating it. (In our own experience, we have minimised this problem by using an enzyme cleaner on the probe after use.)
In summary, our results indicate that although a capsulorhexis produced by diathermy has considerable elasticity, it is significantly less elastic than a continuous tear capsulorhexis, which demonstrated an average $60 \%$ elasticity in accordance with previous reports. Furthermore, the diathermy capsulorhexis has less predictable elastic behaviour when being stressed. Our results also support the view that a CTCC is compatible with nuclear expression with the appropriate technique, but a diathermy capsulorhexis has a higher chance of radial tear formation than a CTCC following nuclear delivery. These differences are due to the nature of the capsular edge following diathermy, which shows gross morphological differences to the edge of a CTCC, as seen with the SEM. Therefore, although diathermy has advantages over CTCC in certain clinical situations, we would not advocate its use in circumstances where a torn capsulorhexis is feasible.

The authors would like to thank the Department of Medical Physics at the General Infirmary at Leeds for their help with construction of the calipers, and also the staff of the Eye Bank at the UKTS, Bristol, for providing the eyes used in the study

The electron microscopy was carried out by Dr Ashley Wilson from the Centre for Cell and Tissue Research at the University of York, and was made possible by support from the Special Trustees of the General Infirmary at Leeds.

1 Gimbel HV, Nuehann T. Development, advantages and methods of the continuous circular capsulorhexis technique. 7 Cataract Refract Surg 1990; 16: 31-7.

2 Thim K, Krag S, Corydon L. Stretching capacity of capsulorhexis and nucleus delivery. F Cataract Refract Surg 1991; 17: 27-30.

3 Pande M. Continuous curvilinear (circular) capsulorhexis and planned extracapsular cataract extraction - are they complanned extracapsular cataract extraction

$4 \mathrm{Krag} S$, Thim K, Corydon L. Strength of the lens capsule during hydroexpression of the nucleus. $\mathcal{F}$ Cataract Refract Surg 1993; 19: 205-8.

5 Klöti R. Bipolar-Nassfeld-Diathermie in der Mikrochirugie. Klin Monatsbl Augenheilkd 1984; 442: 184

6 Gassmann F, Schimmelpfennig B, Klöti R. Anterior capsulotomy by means of bipolar radio-frequency endodiathermy. 7 Cataract Refract Surg 1988; 14: 673 .

7 Assia EI, Apple DJ, Tsai JC, Lim ES. The elastic properties of the lens capsule in capsulorhexis. Am $\mathcal{F}$ Ophthalmol 1991; 111: 628-32.

8 Gimbel H. Two-stage capsulorhexis for endocapsular phacoemulsification. F Cataract Refract Surg 1990; 16: 246-9.

9 Koch DD, Liu JF. Zonular encroachment on the anterior capsular zonular-free zone. Am $\mathcal{F}$ Ophthalmol 1988; 106: 491-2.

10 Assia EI, Apple DJ, Tsai JC, Morgan RC. Mechanism of radial tear formation and extension after anterior capsulectomy. Ophthalmology 1991; 98: 432-7.

11 Davison JA. Analysis of capsular bag defects and intraocular lens positions for consistent centration. $₹$ Cataract Refract Surg 1986; 12: 124-9.

12 Fisher RF. Elastic constants of the human lens capsule. IPhysiol 1969; 201: 1 .

13 Fisher RF. The influence of age on some ocular basement membranes. Eye 1987; 1: 184.

14 Thim K, Krag S, Corydon L. Hydroexpression and viscoexpression of the nucleus through a continuous circular capsulorhexis. $\mathcal{F}$ Cataract Refract Surg 1993; 19: 209-12.

15 Farnsworth PN, Mauriello JA, Burke-Gadomski P, Kulyk T, Cinnoti AA. Surface ultrastructure of the human lens capsule and zonular attachments. Invest Ophthalmol 1976; 15: $36-40$.

16 Barnard K, Burgess SA, Carter DA, Woolley DM. Threedimensional structure of type IV collagen in the mammalian lens capsule. F Struct Biol 1992; 108: 6-13.

17 Straatsma BR, Lightfoot DO, Barke RM, Horwitz J. Lens capsule and epithelium in age-related cataract. Am $\mathcal{F}$ Ophthalmol 1991; 112: 283-96.

18 Marshall GE, Konstas AG, Bechrakis NE, Lee WR. An immunoelectron microscope study of the aged human lens capsule. Exp Eye Res 1992; 54: 393-401

19 Marshall J, Beaconsfield M, Rothery S. The anatomy and development of the human lens and zonules. Trans Ophthalmol Soc UK 1982; 102: 423-40. 